\title{
A Comparative Study of Analytical Solutions to the Coupled Van-der-Pol's Non-linear Circuits Using the He's Method (HPEM) and (BPES)
}

\author{
Hüseyin Koçak ${ }^{1}$, Ahmet Yıldırım ${ }^{1}$, Dahong Zhang ${ }^{2}$, Karem Boubaker ${ }^{3}$, Syed Tauseef Mohyud-Din ${ }^{4}$ \\ ${ }^{1}$ Department of Mathematics, Ege University, Bornova-İzmir, Turkey \\ ${ }^{2}$ Department of Physics, South China University, Guangzhou, China \\ ${ }^{3}$ École Supérieure des Sciences et Techniques de Tunis, University of Tunis, Tunis, Tunisia \\ ${ }^{4}$ Department of Basic Sciences, HITEC University, Taxila Cantt, Pakistan \\ E-mail:hkocak.ege@gmail.com,ahmet.yildirim@ege.edu.tr, \\ Received October 6, 2010; revised May 13, 2011; accepted May 20, 2011
}

\begin{abstract}
In this paper, the He's parameter-expanding method (HPEM) and the 4q-Boubaker Polynomials Expansion Scheme (BPES) are used in order to obtain analytical solutions to the non-linear modified Van der Pol's oscillating circuit equation. The resolution protocols are applied to the ordinary Van der Pol equation, which annexed to conjoint delayed feedback and delay-related damping terms. The results are plotted, and compared with exact solutions proposed elsewhere, in order to evaluate accuracy.
\end{abstract}

Keywords: Van-der-Pol's Oscillating Circuit, Delayed Feedback, Damping, BPES, HPEM, Exact Solutions, Electrical Triode-Valve Circuit

\section{Introduction}

Originally, the Van der Pol's equation was associated, in the 1920 s, with an electrical triode-valve circuit (Figure 1). In the last decades' literature, it was the subject of several investigations due to the panoply of dynamical oddness as relaxation oscillations, elementary bifurcations, quasiperiodicity, and chaos. Its application has already reached nerve pulse propagation and electric potential evolution across neural membranes.

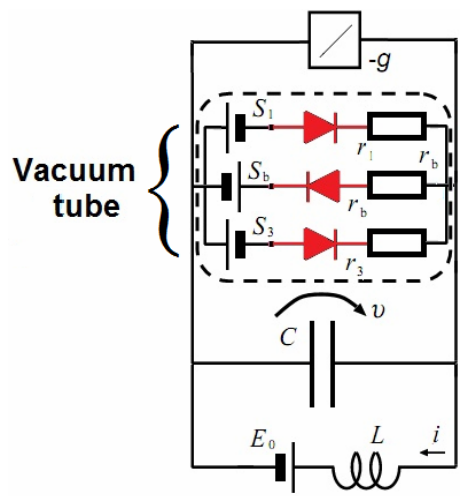

Figure 1. Van der Pol oscillator synoptic scheme.
The actual study tries to give a theoretical supply to the recent attempts to yield analytical solutions to this equation, like the studies of D. D. Ganji et al. [1,2] and A. Rajabi et al. [3] in the heat transfer domain, the investigations of L. Cveticanin [4] and J. H. He [5-7] on nonlinear mechanics, fluid dynamics and oscillating systems modelling (Figure 2).

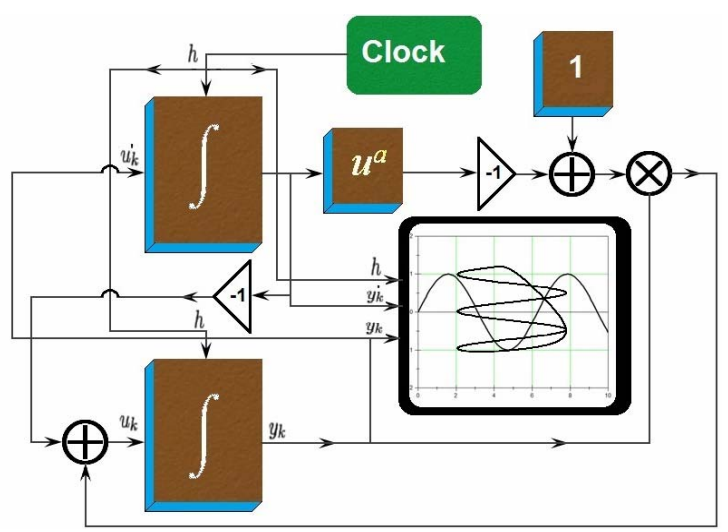

Figure 2. A prototype of Van der Pol oscillating systems modelling (The two integrators are Trapezoidal-type $\left.\left\{y_{k}=h / 2\left(u_{k}+u_{k-1}\right)+y_{k-1}\right\}\right)$. 
Among the different formulations, the well-known standard boundary value-free Van der Pol oscillator problem (BVFP) is given by F. M. Atay [8] by the following system (1):

$$
\left\{\begin{array}{l}
x^{\prime \prime}(t)+x(t)=\varepsilon \times f\left(x(t), x^{\prime}(t), x(t-\tau)\right) \\
f\left(x(t), x^{\prime}(t), x(t-\tau)\right)=\left(1-x(t)^{2}\right) x^{\prime}(t)+k \times x(t-\tau)
\end{array}\right.
$$

where $\tau$ is a positive parameter representing the delay, $\varepsilon>0$ and $k$ is the feedback gain.

A simpler formulation is that of W. Jiang et al. [9]:

$$
\left\{\begin{array}{l}
\dot{x}(t)=y(t) \\
\dot{y}(t)=-x(t)+k f(x(t-\tau))-\varepsilon \times\left(x(t)^{2}-1\right) y(t)
\end{array}\right.
$$

In this study, an attempt to give analytical solution to the nonlinear second-order Van der Pol equation annexed to conjoint delayed feedback and delay-related damping terms as presented by A. Kimiaeifar et al. [10]:

$$
\left\{\begin{array}{l}
\dot{x}(t)=y(t) \\
\dot{y}(t)=-x(t)-\varepsilon \times\left(1-x(t)^{2}\right) y(t)+k \times x(t-\tau) \\
x(0)=x_{0}=H \neq 0 \\
x^{\prime}(0)=x_{0}^{\prime}=0
\end{array}\right.
$$

\section{Analytical Solutions Derivation}

\subsection{The Enhanced He's Parameter-Expanding Method (HPEM) Solution}

The resolution protocol based on the enhanced He's parameter-expanding method (HPEM) is founded on the infinite serial expansions:

$$
\left\{\begin{array}{l}
x(t)=\sum_{n=0}^{+\infty} \varepsilon^{n} x_{n}(t) \\
x(t-\tau)=\sum_{n=0}^{+\infty} \varepsilon^{n} x_{n}(t-\tau)
\end{array}\right.
$$

Substituting these expansions in the main equation Equation (3) and processing with the standard perturbation method, it has been demonstrated [10] that a solution of the kind:

$$
x_{0}(t)=H \cos (\omega \times t+\phi)
$$

where $H, \omega$ and $\phi$ are constant, gives:

$$
\begin{aligned}
& \ddot{x}_{1}(t)+H \omega \sin (\omega \times t+\phi)+\omega^{2} x_{1}(t)+v_{1} H \cos (\omega \times t+\phi) \\
& -H^{3} \omega \cos ^{2}(\omega \times t+\phi) \sin (\omega \times t+\phi) \\
& -k H \cos (\omega \times t+\phi) \cos (\omega \times \tau+\phi) \\
& -k H \sin (\omega \times t+\phi) \sin (\omega \times \tau+\phi)=0
\end{aligned}
$$

with, as a final solution (Equation (4d)):

\subsection{The Boubaker Polynomials Expansion Scheme (BPES)-Related Solution}

The resolution protocol is based on the Boubaker polynomials expansion scheme (BPES) [11-23]. The first step of this scheme starts by applying the expressions:

$$
x(t)=\frac{1}{2 N_{0}} \sum_{k=1}^{N_{0}} \lambda_{k} \times B_{4 k}\left(t \times r_{k}\right)
$$

where $B_{4 k}$ are the $4 \mathrm{k}$-order Boubaker polynomials, is the normalized time $(t \in[0,1]), r_{k}$ are $B_{4 k}$ minimal positive roots, $N_{0}$ is a prefixed integer, and $\left.\lambda_{k}\right|_{k=1 . . N_{0}}$ are unknown pondering real coefficients.

Consequently, it comes that:

$$
y(t)=\dot{x}(t)=\frac{1}{2 N_{0}} \sum_{k=1}^{N_{0}} \lambda_{k} r_{k} \times \frac{\mathrm{d} B_{4 k}\left(t \times r_{k}\right)}{\mathrm{d} t}
$$

The main advantage of these formulations (Equations (4) and $(5))$ is the fact of verifying the boundary conditions in Equation (3), at the earliest stage of resolution protocol. In fact, due to the properties of the Boubaker polynomials [12-18], and since $\left.r_{k}\right|_{k=1 . . N_{0}} r_{k}$ are the roots of $\left.B_{4 k}\right|_{k=1 . . N_{0}}$, the following conditions stand :

$$
\left\{\begin{array}{l}
\left.x(t)\right|_{t=0}=-\frac{1}{N_{0}} \sum_{k=1}^{N_{0}} \lambda_{k}=A=x_{0} \neq 0 \\
\left.\frac{\mathrm{d} x(t)}{\mathrm{d} t}\right|_{t=0}=\frac{1}{2 N_{0}} \sum_{k=1}^{N_{0}} \lambda_{k} r_{k} \times\left.\frac{\mathrm{d} B_{4 k}\left(t \times r_{k}\right)}{\mathrm{d} t}\right|_{t=0}=0
\end{array}\right.
$$

By introducing expressions (4) and (6) in the system (3), and by majoring and integrating along the interval $[0,1], x(t)$ is confined, through the coefficients $\left.\lambda_{k}\right|_{k=1 . . N_{0}}$, to be a weak solution of the system:

$$
\left\{\begin{array}{l}
\sum_{k=1}^{N_{0}} r_{k}^{2} \lambda_{k} M_{k}+\varepsilon \sum_{k=1}^{N_{0}} r_{k} \lambda_{k} P_{k}-\sum_{k=1}^{N_{0}} \lambda_{k}\left(Q_{k}-k \times R_{k}(\tau)\right)=0 \\
M_{k}=\int_{0}^{1} \frac{\mathrm{d}^{2} B_{4 k}\left(t \times r_{k}\right)}{\mathrm{d} t^{2}} \mathrm{~d} t \\
P_{k}=\int_{0}^{1} \frac{\mathrm{d} B_{4 k}\left(t \times r_{k}\right)}{\mathrm{d} t} \mathrm{~d} t \\
Q_{k}=\int_{0}^{1} B_{4 k}\left(t \times r_{k}\right) \mathrm{d} t \\
R_{k}(\tau)=\int_{0}^{1} B_{4 k}\left((t-\tau) \times r_{k}\right) \mathrm{d} t \\
\sum_{k=1}^{N_{0}} \lambda_{k}=-N_{0} x_{0}=-N_{0} H
\end{array}\right.
$$


The set of solutions $\left.\hat{\lambda}_{k}\right|_{k=1, \cdots, N_{0}}$ is the one which mini mizes, for given values of $\varepsilon$ and $k$ the Minimum Square function $\Psi_{M S}(\varepsilon, k)$ :

$$
\begin{aligned}
& \Psi_{M S}(\varepsilon, k) \\
& =\left(\sum_{k=1}^{N_{0}} r_{k}^{2} \hat{\lambda}_{k} M_{k}+\varepsilon \sum_{k=1}^{N_{0}} r_{k} \hat{\lambda}_{k} P_{k}-\sum_{k=1}^{N_{0}} \hat{\lambda}_{k}\left(Q_{k}-k \times R_{k}(\tau)\right)\right)^{2}(8)
\end{aligned}
$$

under the intrinsic condition:

$$
\sum_{k=1}^{N_{0}} \hat{\lambda}_{k}=-N_{0} H
$$

The condition expressed by Equation (9) ensures a non-zero solution to the system (8). The convergence of the algorithm is tested relatively to increasing values of $N_{0}$.

The correspondent solutions are represented in Figure 3 for the data gathered in Table 1, along with the exact solutions given by F. M. Atay [8] and A. Kimiaeifar et al. [10]. It is noted that F. M. Atay [8] demonstrated that the presence of delay can change the amplitude of limit cycle oscillations, or suppress them altogether through derivative-like effects, while A. Kimiaeifar et al. [10] yielded a highly accurate solution to the same classical Van der Pol equation with delayed feedback and a modified equation where a delayed term provides the damping. The features of the proposed solutions [8-10] (namely behavior at starting phase, first derivatives at limit time, etc.) are concordant with the actually proposed results.

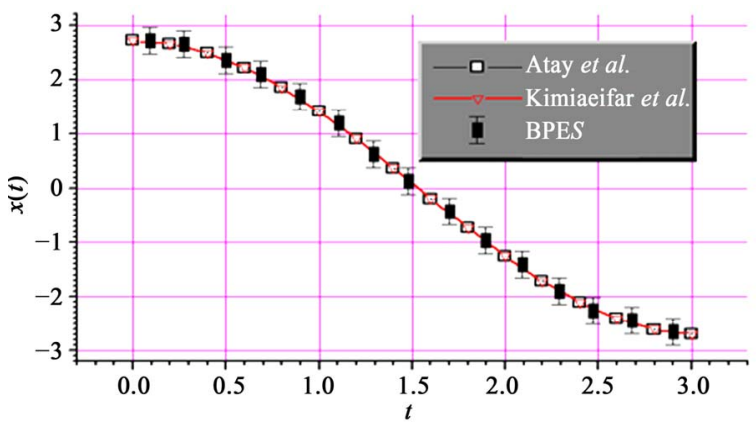

Figure 3. Analytical solutions plots.

Table 1. Solution parameters values.

\begin{tabular}{cc}
\hline Parameter & Value \\
\hline$\varepsilon$ & $\mathbf{0 . 1}$ \\
$k$ & $-\mathbf{1 . 0}$ \\
$\tau$ & $\mathbf{1 . 0}$ \\
$x_{0}$ & $\mathbf{2 . 7 5}$ \\
$\dot{x}_{0}$ & $\mathbf{0 . 0}$ \\
$N_{0}$ & $\mathbf{3 1}$ \\
$\phi$ & $\mathbf{0 . 0}$ \\
\hline
\end{tabular}

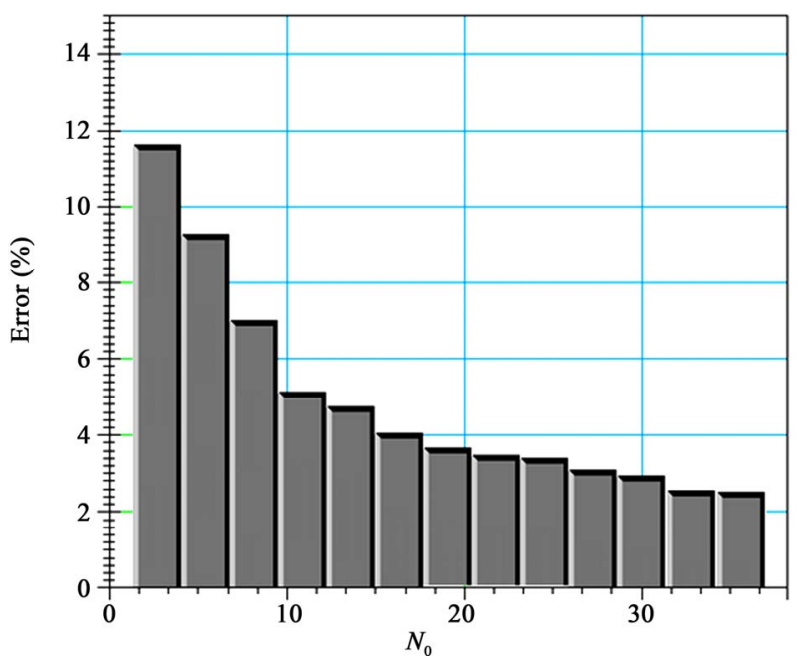

Figure 4. Mean absolute error versus $N_{0}$.

\section{Results and Discussions}

The results show a good agreement between the proposed analytical solutions (Figure 3) and those of the recent studies published elsewhere. The mean absolute error (for $N_{0}>30$ ) was less than $3.33 \%$ (Figure 4). The convergence of the BPES-related protocol has been recorded for the values of $N_{0}$ superior to 30 .

\section{Conclusions}

In this paper, we have used the enhanced He's parameter-expanding Method (HPEM) along with the Boubaker Polynomials Expansion Scheme (BPES) in order to obtain the Van der Pol's characteristic periodical solutions.

The obtained solutions were in acceptable agreement with those obtained from values of similarly performed methods. The typical periodical aspect of the oscillations, already yielded [2,10,24-27] by the enhanced He's parameter-expanding method (HPEM) could be reproduced using a simple and convergent polynomial approximation. This method was based on an original protocol which reduces the stochastic nonlinear system into an equivalent deterministic nonlinear system. This simple and controllable reduction is carried out through the verification of the initial conditions, in the solution basic expression, prime to launching the resolution process.

The results show that the methods are very promising ones and might find wide applications, particularly when exact solutions expressions are difficult to establish [28-34].

\section{References}

[1] D. D. Ganji and A. Rajabi, "Assessment of Homotopy Perturbation and Perturbation Methods in Heat Transfer Radiation Equations," International Communications in 
Heat and Mass Transfer, Vol. 33, No. 3, 2006, pp. 391400. doi:10.1016/j.icheatmasstransfer.2005.11.001

[2] D. D. Ganji and A. Sadighi, "Application of He's Homotopy-Perturbation Method to Nonlinear Coupled Systems of Reaction-Diffusion Equations," International Journal of Nonlinear Sciences and Numerical Simulation, Vol. 7, No. 4, 2006, pp. 411-418.

doi:10.1515/IJNSNS.2006.7.4.411

[3] A. Rajabi, D. D. Ganji and H. Taherian, "Application of Homotopy Perturbation Method in Nonlinear Heat Conduction and Convection Equations," Physics Letters A, Vol. 360, No. 4-5, 2007, pp. 570-573. doi:10.1016/j.physleta.2006.08.079

[4] L. Cveticanin, "Homotopy-Perturbation Method for Pure Nonlinear Differential Equation," Chaos, Solitons \& Fractals, Vol. 30, No. 5, 2006, pp. 1221-1230. doi:10.1016/j.chaos.2005.08.180

[5] J. H. He, "Homotopy Perturbation Method for Bifurcation of Nonlinear Problems," International Journal of Nonlinear Sciences and Numerical Simulation, Vol. 6, No. 2, 2005, pp. 207-208.

[6] J. H. He, "Homotopy Perturbation Method For Solving Boundary Value Problems," Physics Letters A, Vol. 350, No. 1-2, 2006, pp. 87-88.

[7] J. H. He, "Limit Cycle and Bifurcation of Nonlinear Problems," Chaos, Solitons \& Fractals, Vol. 26, No. 3, 2005, pp. 827-833. doi:10.1016/j.chaos.2005.03.007

[8] F. M. Atay, "Van der Pol's Oscillator under Delayed Feedback," Journal of Sound and Vibration, Vol. 218, No. 2, 1998, pp. 333-339. doi:10.1006/jsvi.1998.1843

[9] W. Jiang and J. Wei, "Bifurcation Analysis in Van der Pol's Oscillator with Delayed Feedback," Journal of Computational and Applied Mathematics, Vol. 213, No. 2, 2008, pp. 604-615. doi:10.1016/j.cam.2007.01.041

[10] A. Kimiaeifar, A. R. Saidi, A. R. Sohouli and D. D. Ganji, "Analysis of Modified Vander Pol's Oscillator Using He's Parameter-Expanding Methods," Current Applied Physics, Vol. 10, No. 1, 2010, pp. 279-283. doi:10.1016/j.cap.2009.06.006

[11] J. Ghanouchi, H. Labiadh and K. Boubaker, “An Attempt to Solve the Heat Transfert Equation in a Model of Pyrolysis Spray Using 4q-Order m-Boubaker Polynomials," International Journal of Heat \& Technology, Vol. 26, No. 1, 2008, pp. 49-53.

[12] O. B. Awojoyogbe and K. Boubaker, "A Solution to Bloch NMR Flow Equations for the Analysis of Homodynamic Functions of Blood Flow System Using m-Boubaker Polynomials," Current Applied Physics, Vol. 9, No. 3, 2009, pp. 278-288. doi:10.1016/j.cap.2008.01.019

[13] H. Labiadh and K. Boubaker, "A Sturm-Liouville Shaped Characteristic Differential Equation As a Guide to Establish a Quasi-Polynomial Expression to the Boubaker Polynomials," Differential Equations and Control Processes, Vol. 2, No. 2, 2007, pp. 117-133.

[14] S. Slama, J. Bessrour, M. Bouhafs and K. B. Ben Mahmoud, "Numerical Heat Transfer, Part A: Application," An International Journal of Computation and Methodol- ogy, Vol. 48, No. 6, 2005, pp. 401-404.

[15] S. Slama, M. Bouhafs and K. B. Ben Mahmoud, "A Boubaker Polynomials Solution to Heat Equation for Monitoring A3 Point Evolution During Resistance Spot Welding," International Journal of Heat and Technology, Vol. 26, No. 2, 2008, pp. 141-146.

[16] H. Rahmanov, "Triangle Read by Rows: Row n Gives Coefficients of Boubaker Polynomial B_ $n(x)$, Calculated for $\mathrm{X}=2 \cos (t)$, Centered by Adding $-2 \cos (n t)$, Then Divided by 4, in Order of Decreasing Exponents," OEIS (Encyclopedia of Integer Sequences), A160242.

[17] H. Rahmanov, "Triangle Read by Rows: Row n Gives Values of the 4q-28Boubaker Polynomials B_4q(X) (Named after Boubaker Boubaker (1897-1966)), Calculated for X $=1$ (or -1$)$," OEIS (Encyclopedia of Integer Sequences), A162180.

[18] S. Tabatabaei, T. Zhao, O. Awojoyogbe and F. Moses, "Cut-Off Cooling Velocity Profiling Inside a Keyhole Model Using the Boubaker Polynomials Expansion Scheme," Heat and Mass Transfer, Vol. 45, No. 10, 2009, pp. 1247-1255. doi:10.1007/s00231-009-0493-X

[19] S. Fridjine and M. Amlouk, "A New Parameter: An ABACUS for Optimizig Functional Materials Using the Boubaker Polynomials Expansion Scheme," Modern Physics Letters B, Vol. 23, No. 17, 2009, pp. 2179-2182. doi:10.1142/S0217984909020321

[20] A. Belhadj, J. Bessrour, M. Bouhafs and L. Barrallier, "Experimental and Theoretical Cooling Velocity Profile Inside Laser Welded Metals Using Keyhole Approximation and Boubaker Polynomials Expansion," Journal of Thermal Analysis and Calorimetry, Vol. 97, No. 3, 2009, pp. 911-920. doi:10.1007/s10973-009-0094-4

[21] A. Belhadj, O. Onyango and N. Rozibaeva, "Boubaker Polynomials Expansion Scheme-Related Heat Transfer Investigation Inside Keyhole Model," Journal of Thermophysics Heat Transfer, Vol. 23, No. 6, 2009, pp. 639642.

[22] A. Chaouachi, K. Boubaker, M. Amlouk and H. Bouzouita, "Enhancement of Pyrolysis Spray Disposal Performance Using Thermal Time-Response to Precursor Uniform Deposition," The European Physical Journal Applied Physics, Vol. 37, No. 1, 2007, pp. 105-109. doi:10.1051/epjap:2007005

[23] D. H. Zhang and F. W. Li, “A Boubaker Polynomials Expansion Scheme BPES-Related Analytical Solution to Williams-Brinkmann Stagnation Point Flow Equation at a Blunt Body," Journal of Engineering Physics and Thermophysics, Vol. 84, No. 3, 2009, pp. 618-623.

[24] Z. L. Tao, "Frequency-Amplitude Relationship of Nonlinear Oscillators by He's Parameter-Expanding Method," Chaos, Solitons \& Fractals, Vol. 41, No. 2, 2009, pp. 642-645. doi:10.1016/j.chaos.2008.02.036

[25] L. Xu, "He's Parameter-Expanding Methods for Strongly Nonlinear Oscillators," Journal of Computational and Applied Mathematics, Vol. 207, No. 1, 2007, pp. 148-154. doi:10.1016/j.cam.2006.07.020

[26] D. D. Ganji, M. Rafei, A. Sadighi and Z. Z. Ganji, "A Comparative Comparison of He's Method with Perturba- 
tion and Numerical Methods for Nonlinear Vibrations Equations," International Journal of Nonlinear Dynamics in Engineering and Sciences, Vol. 1, No. 1, 2009, pp. 120.

[27] J. H. He, "Determination of Limit Cycles for Strongly Nonlinear Oscillators," Physical Review Letters, Vol. 90, No. 17, 2003, pp. 1-11. doi:10.1103/PhysRevLett.90.174301

[28] Z. M. Odibat and S. Momani, "Application of Variational Iteration Method to Nonlinear Differential Equations of Fractional Order," International Journal of Nonlinear Sciences and Numerical Simulation, Vol. 7, No. 1, 2006, pp. 27-34. doi:10.1515/IJNSNS.2006.7.1.27

[29] E. Yusufoglu, "Variational Iteration Method for Construction of Some Compact and Non Compact Structures of Klein-Gordon Equations," International Journal of Nonlinear Sciences and Numerical Simulation, Vol. 8, No. 2, 2007, pp. 152-158.

[30] M. D'Acunto, “Self-Excited Systems: Analytical Deter- mination of Limit Cycles," Chaos, Solitons \& Fractals, Vol. 30, No. 3, 2006, pp. 719-724.

doi:10.1016/j.chaos.2006.03.070

[31] J. K. Hale, "Averaging Methods for Differential Equations with Retarded Arguments and a Small Parameter," Journal of Differential Equations, Vol. 2, No. 1, 1966, pp. 57-73. doi:10.1016/0022-0396(66)90063-5

[32] A. Golbabai and D. Ahmadian, "Homotopy Pade Method for Solving Linear and Nonlinear Integral Equations," International Journal of Nonlinear Dynamics in Engineering and Sciences, Vol. 1, No. 1, 2009, pp. 59-66.

[33] J. H. He, "Some Asymptotic Methods for Strongly Nonlinear Equations," International Journal of Modern Physics B, Vol. 20, No. 10, 2006, pp. 1141-1199. doi:10.1142/S0217979206033796

[34] J. H. He, "Book Keeping Parameter in Perturbation Methods," International Journal of Nonlinear Sciences and Numerical Simulation, Vol. 2, No. 3, 2001, pp. 257-264. 